\title{
Simulation network-level relationships of traffic flow
}

\author{
Vladimir Zyryanov ${ }^{1 *}$ \\ ${ }^{1}$ Don State Technical University, 344000 Rostov-on-Don, Russia
}

\begin{abstract}
This paper describes possibilities of the macroscopic fundamental diagram (MFD) of traffic flow to predict the conditions of operation of the road network in urban areas. This study examines relationships between traffic flow parameters on the network level. Microscopic traffic simulation has provided important data on the estimation of road capacity, velocity, trip time, and detection of congestions reasons. Data of spatial distribution density in network useful for implementing approach based on gating policy on subnetwork using MFD. It presents the results of a simulation using the example of central area of Rostov-on-Don
\end{abstract}

\section{Introduction}

The important problem in modern cities is the development of estimation methods of proper operation of an urban network. However, specific character of urban traffic largely limits an opportunity of realization of experiments with the previously given characteristics of a road situation. In these conditions the mathematical modelling in solving road traffic problems is an integral part of research and the considerable advantages can be obtained with the usage of traffic simulation. This is especially important in the research at the network level.

In recent years, there is an increase of relevance of researches on estimating and forecasting of traffic states at the network level for the cities or large areas of the city. Development of this direction has been limited due to the lack of experimental data as the essential saturation of network of transport detectors is required. Therefore, it was difficult to determine how reliable traffic data describing the whole network is. However, with the advent of modern software for traffic simulation and advanced traffic information systems appeared a basis for the development of the use of macro models at the network level.

Analyzing the evolution of the macro models of traffic flow it may be noted that already in 1966 there was an option for the network macro model, including such parameters as the density on the network and the area of the zone under consideration [19]. Then macroscopic fundamental diagram of traffic flow for road network was proposed by J. Godfrey, who investigated interrelations between the average velocity, density and overall mileage of traffic flow on the road network of the city [12]. Approximately, at the same time J. Wardrop suggested a relationship between the speed and flow for any given area with additional data about average street width and the average distance between intersections [20]. Main findings

\footnotetext{
* Corresponding author: tolbaga@mail.ru
} 
that have been obtained as a result of these studies concluded that at the network level there is relationship between the velocity and density as for the macro models on the road segment.

The next stage of the application of macro models at the network level is associated with the appearance of two-fluid model of the kinetic theory of traffic flow [2, 13, 14]. The twofluid model of traffic flow through the explanation state of two parts - the moving and stopped allow to predict the parameters of traffic in urban streets, as well as at the network level.

With the development of simulation, methods and the detection of traffic network data started a study on the use of macro models for such objects. The modern concept of the theory was proposed by Daganzo as theoretical relationship between the accumulation and the exit flow for urban network. The currently existing approach of Macroscopic Fundamental Diagram (MFD) of traffic flow and its parameters have been formed in the researches of Daganzo and Geroliminis [4-7,9-11]. Sometimes in order to highlight the nature of the network approach it is used another term - Network Fundamental Diagram (NFD). This topic of research is important for traffic management and traffic demand management because it is necessary to have information on the acceptable amount of vehicles in the various zones of the network.

Based on the parameters of the MFD, some traffic management algorithms with using ITS technology are suggested. For example, the gating strategy gives a significant improvement of traffic conditions $[8,15]$. According to the simulation results of Chania city network, decrease of traffic delay may be up to $40 \%$.

\section{Methods of analysis network macromodels}

One of the first approaches to the use of network-level macro models was proposed by Smeed in the study of traffic parameters in urban areas. As a result of these studies it was determined the potential number of vehicles on the network to avoid congestion. He took into account the network topology, the width of road, type of intersections, etc. The Smeed function [19] can be formulated as:

$$
N=a f c \sqrt{A}
$$

where $N$ is a number of vehicles per unit time that can enter the city center, $f$ - fraction of area occupied by roads, $c$ - lane capacity and $a$-parameter.

Wardrop [20] proposed a model for the calculation of the tempo of driving in the network, taking into account the distance between intersections:

$$
\frac{1}{v}=\frac{1}{\alpha(1-q / c)}+\frac{f b}{1-q / \lambda s}
$$

where $v$ - is the average speed, $q$ - traffic volume, $c$ capacity, $s-$ is the saturation flow, $\lambda$ - ratio green time to cycle time, $f$ - the number of signalized intersections per unit of road length, $\alpha$ and $b$ - parameters.

Two-fluid models also can be used for an estimation of a traffic flow in very wide range of road capacity and network topology $[16,17,18,21,22]$. One of the basic advantages of two-fluid models is the usage of parameters to an estimation of trip time structure and a fraction of simultaneously stopping vehicles in an urban network.

Basic equation of two-fluid concept showed the relationship between stop time and trip time:

$$
t_{s}=t-t_{m}^{\frac{1}{n+1}} t^{\frac{n}{n+1}}
$$




$$
f_{s}=1-\left(\frac{t_{m}}{t}\right)^{1 / n+1}
$$

where $t$ - total travel time per unit distance; $t_{r}$ - running time per unit distance; $t_{m}$ - average minimum trip time per unit distance; $f_{s}$ - fraction of stopped vehicles and $n$-indicator.

Using obtained from the kinetic theory the relationship between the density, fraction of the stopped vehicles and specific indicators of travel time and taking in account a generalized model of Pipes for the relationship between speed and density, one can get the following expression for calculating traffic parameters:

$$
\begin{aligned}
& k=k_{j}\left[1-\left(\frac{v}{v_{f}}\right)^{1 / n+1}\right]^{1 / p} \\
& q=v k_{j}\left[1-\left(\frac{v}{v_{f}}\right)^{1 / n+1}\right]^{1 / p}
\end{aligned}
$$

where, $q$ - traffic volume; $k$ - traffic density; $v$-speed; $v_{f}$ - free flow speed; $p$-parameter quality of the traffic network.

Smaller values of the coefficient $n$ (0.6-1) leads to an almost linear relationship between the density and speed of traffic flow. The increase of this coefficient changes the shape of relation $v=f(k)$ resulting in a sharp decrease of speed with increasing traffic density. Model parameter $p$ allows to predict the state of traffic on sections of the road network, with different capacity. Increasing values of the parameter $p$ corresponds to increase capacity and density for a given capacity.

The dynamics of the traffic in the parameters of the macroscopic fundamental diagram is described on the assumption that the accumulation level of cars on the network will influence on the performance of network $[4,5]$ :

$$
\frac{d n(t)}{d t}=f(t)-O(n(t))
$$

where $n$-number vehicles in the network, $f$-input traffic, $O$-output traffic and $t$-time.

The main parameters to describe the MFD are vehicle accumulation in a network, travel production and performance.

Accumulation is a number of vehicles in the network. This parameter can be calculated by multiplying density at the link of network:

$$
A=\sum_{i \in z} k_{i} l_{i}
$$

where $A$ accumulation, $k$ traffic density, $l$ length of the network section and $i$ section number

Travel production is a distance travelled by all vehicles in the network over a unit of time:

$$
P=\sum_{i \in z} q_{i} l_{i}
$$

where $P$ travel production and $q$ traffic volume.

Performance is an average flow in the network: 


$$
E=\frac{\sum_{i \in z} q_{i} l_{i}}{\sum_{i \in z} l_{i}}
$$

where E performance.

The main function of the MFD is to estimate traffic conditions in the network at a different level of accumulation cars in the network. Typically, in graphical form the MFD is performed in "performance - accumulation" coordinates, although there may be other options using the parameters of efficiency and average density.

Ideas of using MFD have been confirmed based on experimental data obtained in different cities. Widely known results obtained in Yokohama (Japan) based on the stationary detectors data and taxis as probe cars. Research area included $10 \mathrm{~km} 2$ in the center of the city. The feature of the road network in this area of the city is a short distance between the intersections, from 100 to $300 \mathrm{~m}$. However, there are also highways passing through the area. Results of this investigation show that an MFD exists for different traffic demand, therefore parameters MFD can be used to demand management and improve accessibility. Then, the experimental study the conditions for obtaining the MFD have been carried out on the road network in Toulouse (France) [3]. It may be highlighted that in these studies it was found that the location of the detector has a significant impact on the shape and scatter of the primary values of the parameters the MFD.

Therefore, there is great interest in applying these approaches to different cities: some scenarios for San Francisco, area around the Nanning International Exhibition Center (China), Chania (Greece), simulation for congested urban network in Yokohama (Japan), Toulouse (France), Amsterdam Ring Road (Netherlands) etc. In fact, all of investigations gives improvement of main mobility parameters - travel time, stop time, mean space speed.

The results of the application of the MFD for downtown San Francisco are widely known. For one of scenarios with three area of perimeter control the travel time and space-mean speed are improved in average $11.7 \%$ and $15.4 \%$ respectively [1]. In case of simulation for strategy of perimeter control in downtown of San Francisco with additional option of dynamic traffic assignment, some of traffic parameters improvement in average $45 \%$ (figure 1a).

In example for Nanning International Exhibition Center, the controlled region included 40 intersections and 13 of them are selected for perimeter control on the base of MFD. During congestion those 13 intersections can be decreased the traffic inflow to regions and keep stable traffic conditions. An average speed increase of almost $12 \%$ results for the whole network and some sections even get about 50\% improvements in compare to scenario without perimeter control (figure1b). Simulation area in Chania (Greece) included 165 links and 8 intersections as gaiting. Results of this study for estimation of gating strategy shows that trip time in simulating area decreased on $40 \%$.

These results are great importance and proves that concept of MFD can be utilized for novel control strategies to improve urban mobility. 


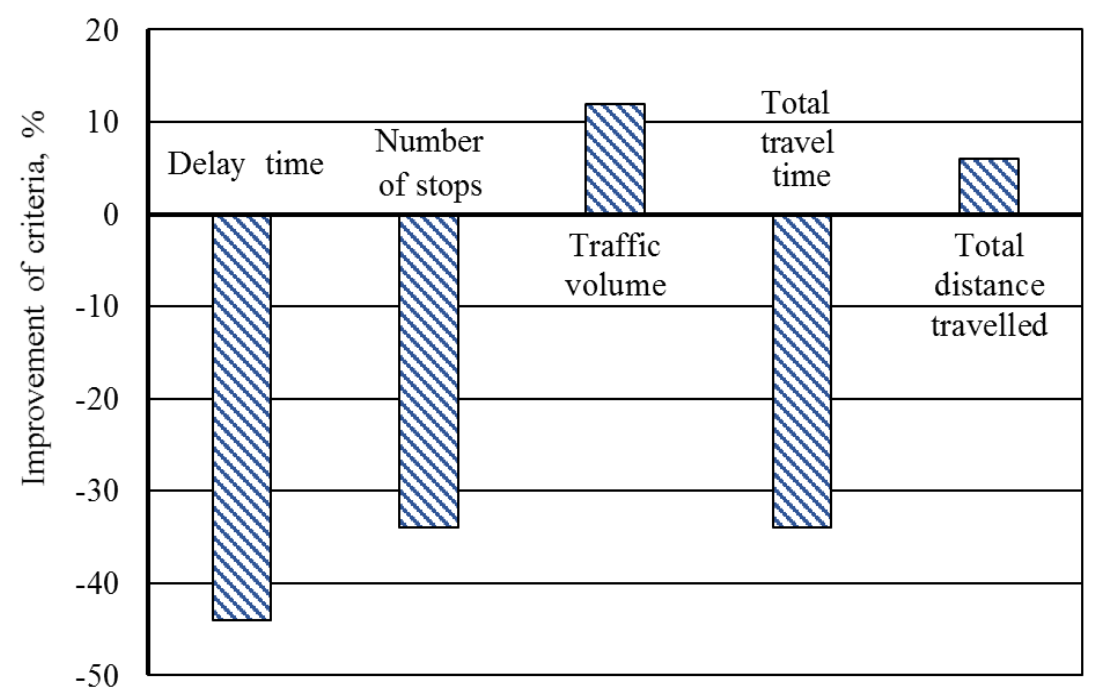

a) downtown San Francisco;

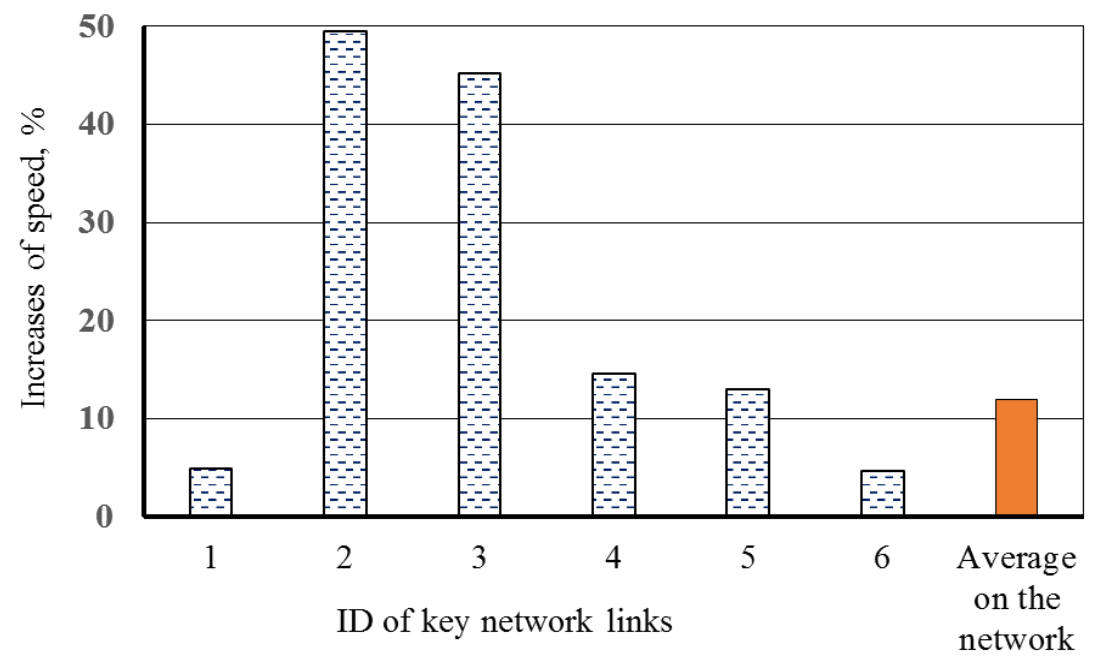

b) Nanning International Exhibition Centre

Fig. 1. Performance parameter for traffic perimeter control.

\section{Results of traffic flow simulation}

Saturation of an urban network by traffic detectors gives it possible to apply MFD for traffic control. These approaches use gaiting policy and perimeter traffic control for temporally reduce the incoming traffic flow to certain areas. These features provide new opportunities for traffic network management. The main information with this approach is such parameters of the MFD as accumulation and travel production. The main advantages in this case are the ability to use active traffic management based on significantly less information. But one need using traffic simulation for adaptive determination of subnetwork for traffic control and determination parameters of MFD for every subnetwork.

According to the traffic control on the base of MFD must be solved following main tasks:

- Collect real-time traffic data for MFD in area of traffic management; 
- Define area of perimeter control;

- Apply of perimeter control strategy.

The overall structure of these tasks is shown in Figure 2.

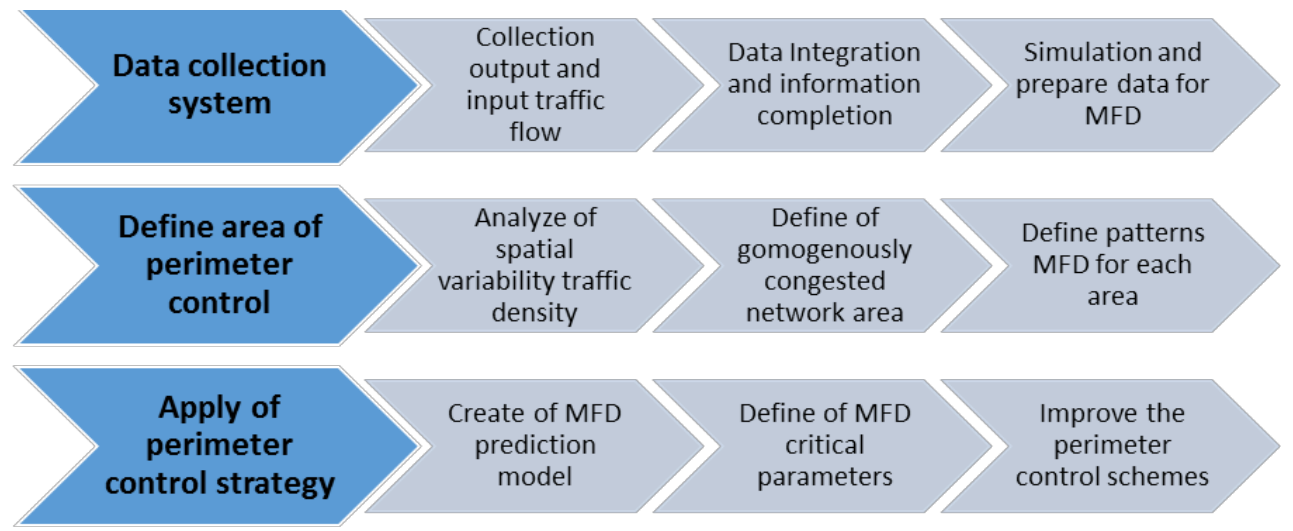

Fig. 2. Main stages application MFD for perimeter control.

In accordance with figure 2 for perimeter control very important MFD in given condition. For study the MFD we used simulation data of a fragment of the central part of Rostov-onDon network. Within the framework of the research part on the basis of the AIMSUN the model of transport network has been created. The total length of the streets in this part of the network is $39 \mathrm{~km}$. As a result of simulation we obtained relationship between travel production, accumulation and performance (fig. 3).

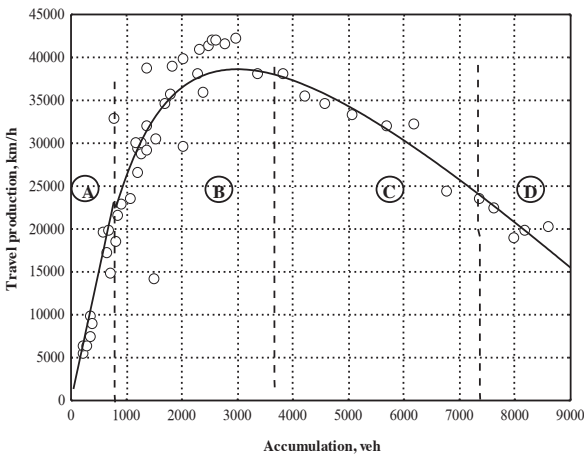

a)

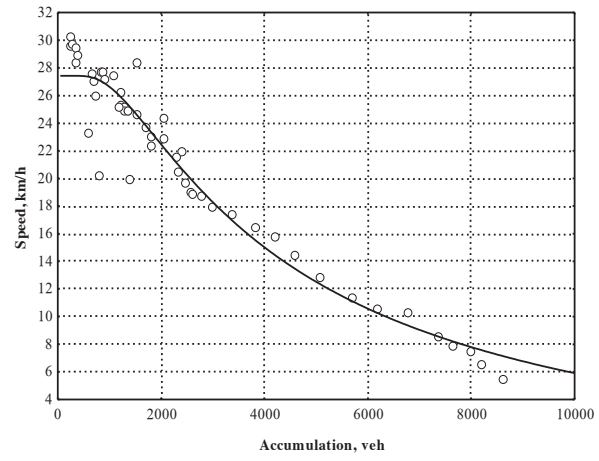

b)

Fig. 3. Relation accumulation vs travel production (a) and average network speed (b)

Relation between accumulation and travel production can be formulated as:

$$
P=A v_{f}\left\{1-\exp \left[1-\left(1+\frac{v_{j}}{n v_{f}}\left(1-\frac{A_{j}}{A}\right)\right)^{n}\right]\right\}
$$

where $P$ - travel production, $A$ - accumulation, $A_{j}-$ maximum accumulation, $v_{f}-$ free flow speed and $n$ parameter.

For a given area the maximum of performance is achieved at the level of accumulation of about 2,500 cars and $10 \%$ of the maximum deviation of the parameter is observed in the range of 2,000 to 3,500 vehicles. This data in the shape of MFD corresponds to the typical 
parameters of the macroscopic fundamental diagram. On this MFD 4 states of the network can be identified. Condition A corresponds to stable state, with an average speed not lower than $25 \mathrm{~km} / \mathrm{h}$. The performance at the state B reaches the maximum, but the speed drops to $17 \mathrm{~km} / \mathrm{h}$. In state $\mathrm{D}$ there is a falling rate of up to $7 \mathrm{~km} / \mathrm{h}$, due to the congestion. At state D performance drops to practically zero. It is very difficult to eliminate this state, even with a substantial restriction of traffic demand. The simulation results show that the length of sections of the network, their capacity, the free flow speed and type of control on the intersections significantly affect the shape of the MFD.

To determine the network area based on perimeter control, it is very important to select the boundaries of the area. One of the main conditions for solving this problem is the choice of the network area in which the variance of the traffic density is lower. When study the MFD properties it is generally accepted the hypothesis of a homogeneous distribution of traffic flow on the network although in fact it depends on the traffic demand and network topology. Nevertheless, in many studies this concept is used. In our studies, we tried to determine the type of spatial vehicles distribution in a network at the different traffic demand.

In contrast to the results of other studies, the hypothesis of a uniform distribution of traffic density on the network was not confirmed in the simulation. For free and stable conditions, the density distribution in the network follows an exponential distribution. For high level average flow density is not obtained statistically significant density distribution on the network sections (figure 4).

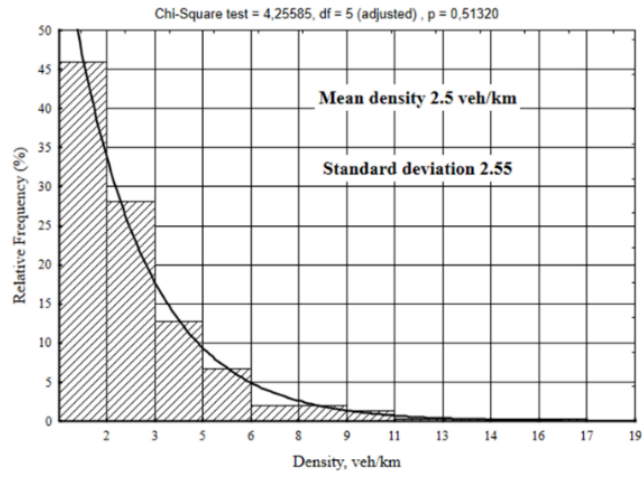

a)

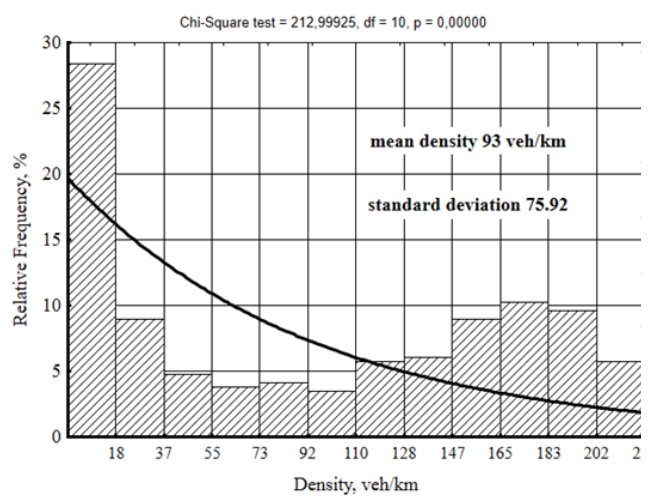

c)

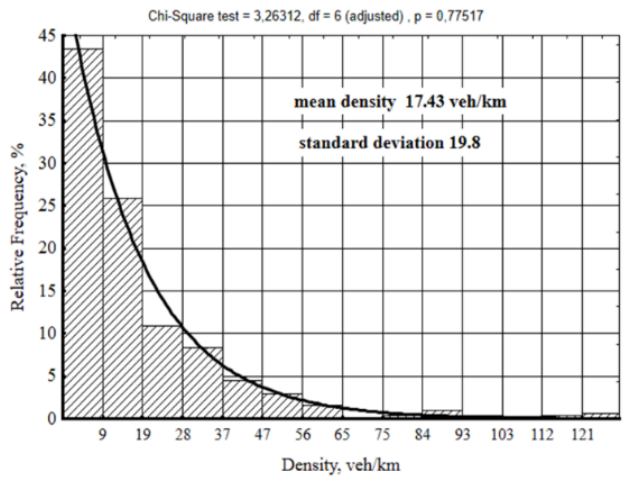

b)

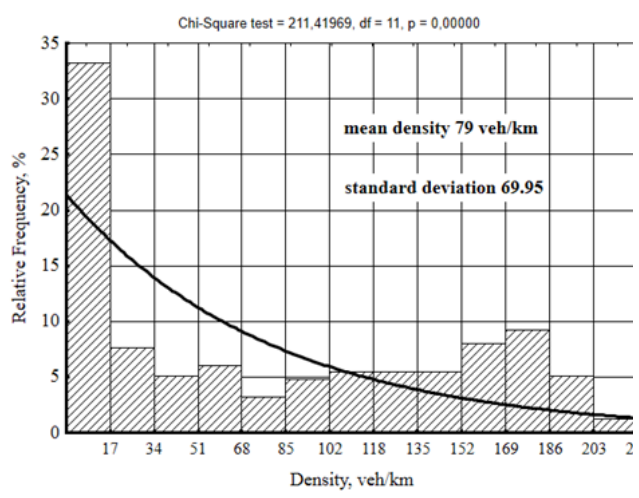

d)

Fig. 4. Spatial distribution density in the network for different traffic state

On one hand, there is considerable number of links with a low density as there is a limited access of cars to those sections in congestion. But on other hand, for very high values of 
density (more than $80 \mathrm{veh} / \mathrm{km}$ ) state of gridlock appears and links with congestion increase. Thus, the spatial density distribution and heterogeneity of the network depend not only on the topology of the network, but also on the traffic volume. It is important in case of perimeter control strategy on the base of MFD for partition the network to define some of homogeneous regions with smaller variances of link densities.

A solution for perimeter control on the base of MFD is to partition the network to define some of homogeneous areas with smaller variances of link densities. This information is important to create well-defined MFD and determine area for perimeter control.

\section{Conclusion}

In this paper we analyzed tendency development macro model of traffic flow. Macroscopic Fundamental Diagram describes the relationship between accumulation, travel production and performance. MFD is very important to understanding of state traffic flow on the network level. We have investigated spatial distribution traffic density on the links of the road network. At traffic density of up to $15-20 \mathrm{veh} / \mathrm{km}$ per lane, the density distribution over road network sections corresponds to an exponential distribution. The coefficient of variation is about $100 \%$ which is typical for this distribution. Under these conditions, the parameters of the traffic flow are homogeneous and guarantee the obtain of a MFD. With an increase in network load, the range of density variation in network sections increases. The density distribution over the network sections is in fact a bimodal one and is not described by classical probability distributions. For these conditions, additional filtering is necessary to obtain a stable shape of MFD. This approach can be used in prediction congestion, estimation of capacity, perimeter traffic control on the base MFD and spatial distribution density in the network.

\section{References}

1. K. Aboudolas, N. Geroliminis, Perimeter and boundary flow control in multi-reservoir heterogeneous networks, Transportation Research, Part B, 55, pp. 265-281 (2013)

2. S. Ardekani, R. Herman, Urban network-wide traffic variables and their relations, Transportation Science, 21 (1), pp. 1-16. (1987)

3. C. Buisson, C. Ladier, Exploring the impact of homogeneity of traffic measurements on the existence of macroscopic fundamental diagrams. Transportation Research Record: Journal of the Transportation Research Board, 2124, pp.127-136 (2009)

4. C.F. Daganzo, Urban Gridlock: Macroscopic modeling and mitigation approaches, Transportation Research Part B, 41 (1), pp. 49-62 (2007)

5. C.F. Daganzo, N. Geroliminis, An analytical approximation for the macroscopic fundamental diagram of urban traffic, Transportation Re-search Part B, 42, pp. 771781 (2008)

6. J. M. Del Castilio, F. G. Benitez, On the functional form of the speed-density relationship - I: General theory. Transp. Res. 29 B. №5. pp. 373-389 (1995)

7. J.M. Del Castilio, F.G. Benitez, On the functional form of the speed-density relationship - II: Empirical investigation. "Transp. Res.", 29 B. №5. pp. 391-406 (1995)

8. V. Dinopoulou, Applications of the urban traffic control strategy TUC, European Journal of Operational Research, 175, No. 3, pp. 1652 - 1665 (2006) 
9. V. Gayah, C.F. Daganzo, Clockwise hysteresis loops in the Macroscopic Fundamental Diagram: An effect of network instability, Transportation Research Part B 45 (4), pp. 643-655 (2011)

10. N. Geroliminis, C.F. Daganzo, Existence of urban-scale macroscopic fundamental diagrams: Some experimental findings, Transportation Research Part B 42, pp. 759770. (2008)

11. N. Geroliminis, J. Sun, Properties of a well-defined macroscopic fundamental diagram for urban traffic, Transportation Research Part B 45, pp. 605-617 (2011)

12. J.W. Godfrey, The mechanism of a road network, Traffic Engineering and Control, 11, pp. 323-327 (1969)

13. D. Helbing, Derivation of a fundamental diagram for urban traffic flow, The European Physical Journal B 70 (2), pp. 229-241 (2009)

14. R. Herman, I. Prigogine, A two - fluid approach to town traffic, "Science", 204, pp. 148-151 (1979)

15. M. Keyvan-Ekbatani, M. Papageorgiou, I. Papamichail, Perimeter Traffic Control via Remote Feedback Gating, Procedia - Social and Behavioral Sciences, 111, 5 February 2014, Pages 645-653 (2014)

16. H. Mahmassani, J. C. Williams and R. Herman, Investigation of Network-Level Traffic Flow Relationships: Some Simulation Results, In Transportation Research Record 971, TRB, National Research Council, Washington, D.C., pp. 121-130 (1984)

17. G.A. Mendez, L.R. da Silva, H.J. Herrmann, Traffic gridlock on complex networks, Physica A: Statistical Mechanics and its Applications, 391 (1), 362-370 (2012)

18. Y. Ji, W. Daamen, S. Hoogendoorn, S. Hoogendoorn-Lanser, and X. Qian, Macroscopic fundamental diagram: investigating its shape using simulation data, Transportation Research Record, Journal of the Transporation Research Board, 2161 pp. 42-48., 2010 (2010)

19. R. J. Smeed, Road Capacity of City Centers, Traffic Engineering and Control, 8 (7), Pages 455-458 (1966)

20. J. G. Wardrop, Journey Speed and Flow in Central Urban Areas, Traffic Engineering and Control, 9 (11), 528-532 (1968)

21. J. C. Williams, H. S. Mahmassani, and R. Herman, Sampling Strategies for Two-Fluid Model Parameter Estimation in Urban Networks, Transportation Research Part A, 29 (3), pp. 229-244 (1995)

22. X. Wu, H. Liu and N. Geroliminis An empirical analysis on the arterial fundamental diagram, Transportation Research Part B: Methodological, 45 (1), pp. 255 - 266 (2011) 